\title{
HELECHOS Y LICOFITOS DE LA RESERVA NATURAL EDUCATIVA COLONIA BENÍTEZ (CHACO, ARGENTINA)
}

\author{
BRUNO DEMATTEIS ${ }^{1} \&$ ESTEBAN I. MEZA-TORRES ${ }^{1}$
}

Summary: Dematteis, B. \& E. I. Meza-Torres. 2015. Ferns and Lycophytes of Colonia Benítez (Chaco, Argentina) Educational Nature Reserve. Bonplandia 24(1): 27-35.

The Educational Nature Reserve of Colonia Benítez comprises approximately 8 ha and is located in the department of Primero de Mayo of the Chaco Province, Argentina. This protected area occurs within the Chaco Oriental District of the Chaco phytogeographic province. The main aims of the present study were to realize an inventory of ferns and lycophytes and provide a key to identify all the taxa present in the reserve. We analyzed the collections deposited at the herbarium CTES and collected new specimens. A total of 12 genera and 17 species were recorded. Doryopteris is the best represented genus with three species: $D$. concolor, $D$. lorentzii and $D$. pentagona. Three genera included two species: Adiantopsis ( $A d$. chlorophylla and Ad. tweediana), Anemia (An. simplicior and An. tomentosa var. anthriscifolia), and Pteris ( $P$. denticulata var. tristicula and $P$. vittata). The remaining genera included only one species: Adiantum lorentzii, Anogramma chaerophylla, Azolla cristata, Hemionitis tomentosa, Microgramma vaccinifolia, Pityrogramma calomelanos var. calomelanos, Pleopeltis minima, and Selaginella sellowii. The terrestrial habit was the best represented with 12 species, one of which was rupicolous and other three were facultative or accidental epiphytes. Only two taxa were strict epiphytes. Azolla cristata was the only aquatic species recorded in the area. Ecological observations and biogeographical analyses are provided for the recorded species.

Key words: Conservation, diversity, floristic inventory, monilophyta, protected area.

Resumen: Dematteis, B. \& E. I. Meza-Torres. 2015. Helechos y licofitos de la Reserva Natural Educativa Colonia Benítez (Chaco, Argentina). Bonplandia 24(1): 27-35.

La Reserva Natural Educativa Colonia Benítez cuenta con una superficie aproximada de ocho ha, está ubicada en el departamento Primero de Mayo, en la provincia del Chaco, Argentina. Desde el punto de vista fitogeográfico, esta área protegida se localiza dentro del Distrito Chaqueño Oriental de la Provincia Chaqueña. Como objetivos de este trabajo se planteó realizar un inventario de helechos y licofitos y una clave para el reconocimiento de los taxa que crecen en esta reserva. A tal fin, se examinaron las colecciones depositadas en CTES, y se realizaron nuevas recolecciones de especímenes. Se registraron 12 géneros con un total de 17 especies, siendo Doryopteris el género mejor representado con tres especies: $D$. concolor, $D$. lorentzii y $D$. pentagona. El género Adiantopsis presentó dos especies ( $A$. chlorophylla y $A$. tweediana), al igual que Anemia con ( $A$. simplicior y $A$. tomentosa var. anthriscifolia) y Pteris

\footnotetext{
${ }^{1}$ Instituto de Botánica del Nordeste, Consejo Nacional de Investigaciones Científicas y Técnicas, Universidad Nacional del Nordeste, CONICET-UNNE, (C.C. 209) 3400, Corrientes, Argentina. E- mail: bdematteis1993@gmail.com; mezatorresii@yahoo.com.ar
} 


\begin{abstract}
(Pteris denticulata var. tristicula y Pteris vittata). Los demás géneros presentaron sólo una especie: Adiantum lorentzii, Anogramma chaerophylla, Azolla cristata, Hemionitis tomentosa, Microgramma vaccinifolia, Pityrogramma calomelanos var. calomelanos, Pleopeltis minima y Selaginella sellowii. El hábito terrícola fue el mejor representado con 12 especies, de las cuales una de ellas es rupícola y otras tres se pueden comportar como epífitos facultativos, en tanto que los estrictamente epífitos fueron representados por sólo dos especies. Azolla cristata fue la única especie acuática presente. Se presentan observaciones ecológicas y un análisis biogeográfico de las especies registradas.
\end{abstract}

Palabras clave: Área protegida, conservación, diversidad, inventario florístico, monilófitas

\section{Introducción}

Los helechos y licofitos son considerados como el segundo grupo más grande de plantas vasculares. La mayor riqueza y diversidad de este grupo se encuentra concentrada en los trópicos, aunque su área de distribución se extiende hasta las regiones templado-frías de ambos hemisferios (de la Sota, 1977). En Argentina, se registran 802 taxones (Zuloaga et al., 2008), los mismos se encuentran concentrados en tres centros de diversidad pteridofítica: Centro Subtropical del Noreste de Argentina, Centro Subtropical del Noroeste y Centro Templado del sur de Chile y Argentina (Ponce et al., 2002). La provincia del Chaco se encuentra situada geográficamente entre los centros Noroeste y Noreste y cuenta con un registro de 56 especies de helechos y licofitos (Zuloaga et al., 2008).

La Reserva Natural Educativa (R.N.E.) Colonia Benítez $\left(27^{\circ} 19^{\prime} 5,77^{\prime \prime} \mathrm{S}\right.$ $58^{\circ} 57^{\prime} 2.43$ ”O) es un área protegida que se encuentra bajo la competencia de la Administración de Parques Nacionales, está localizada en el departamento Primero de Mayo, en la provincia del Chaco, Argentina (Fig. 1). La reserva cuenta con una superficie aproximada de 8 ha, la cual se encuentra separada físicamente en dos sectores, uno de 7 ha y otro de 1 ha (Fig. 1). Ambos sectores están dentro del predio correspondiente a la Estación Experimental Agropecuaria Colonia Benítez (INTA). La reserva fue creada a partir de la propuesta impulsada por Augusto
Schulz, con el fin de proteger y conservar la diversidad biológica de esta ecorregión. El área fue designada como reserva natural estricta el 10 de octubre de 1990 bajo el decreto $\mathrm{N}^{\circ} 2149 / 90$ y el 11 de septiembre de 2002 fue recategorizada como Reserva Natural Educativa mediante el decreto $\mathrm{N}^{\circ}$ 1798/2002.

Desde el punto de vista fitogeográfico la reserva se ubica dentro del Dominio Chaqueño -Provincia Chaqueña- Distrito Chaqueño Oriental, la cual se caracteriza por presentar un clima cálido, con temperaturas medias que oscilan entre $20^{\circ}-23^{\circ} \mathrm{C}$ y precipitaciones medias anuales de $1200 \mathrm{~mm}$. Esta región se caracteriza por un tipo de vegetación predominante, el de bosques xerófilos caducifolios, aunque también cabe destacar la presencia de palmares, sabanas, estepas halófilas, entre otros ambientes (Cabrera, 1971). La Reserva se encuentra próxima a los ríos Paraguay y Paraná, los cuales se consideran como formidables corredores biogeográfico. Este es el único sistema fluvial que tiene sus nacientes en ambientes del trópico húmedo y su desembocadura en regiones templadas húmedas (Oakley et al., 2005).

La R.N.E. Colonia Benítez se ubica sobre un antiguo cauce del río Tragadero dentro del cual se distinguen tres ambientes: un relicto de selva con una zona inundable, una zona de quebrachal degradado y un estero (Fig. 2. A-C). Esta área protegida, a pesar de su escaso tamaño, alberga especies características del Chaco Oriental como lo son Aspidosperma 


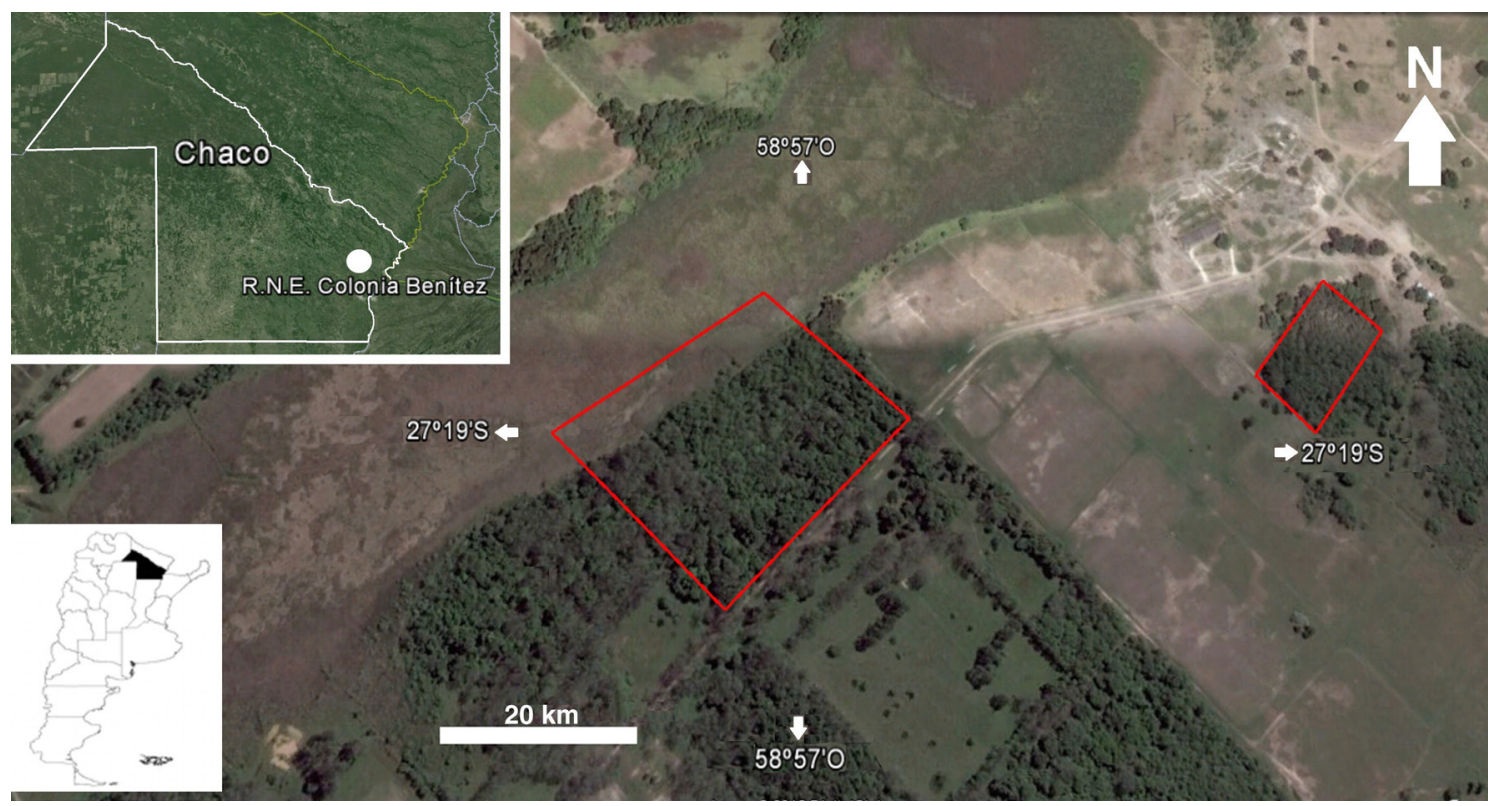

Fig. 1. Ubicación geográfica de la Reserva Natural Educativa Colonia Benítez

quebracho-blanco Schlecht. (quebracho blanco), Caesalpinia paraguariensis (D. Parodi) Burkart (guayacán), Gleditsia amorphoides Griseb. (espina corona), Astronium balansae Engl. (urunday), Diplokeleba floribunda N. E. Br. (ibirá itá), Patagonula americana L. (guayaibí), entre otros (Cabrera, 1971).

A pesar de que el noreste argentino es considerado como zona de concentración de helechos y licofitos (Ponce et al., 2002), son escasos los antecedentes de inventarios de estas plantas en la región. Algunos aportes al conocimiento de la diversidad de helechos y licofitos de esta zona del país son contribuciones limitadas a reservas de la provincia de Misiones (Biganzoli \& Múlgura de Romero, 2004; Márquez et al., 2006; Tressens et al., 2008), y flora para la provincia de Corrientes (Arbo et Tressens, 2002; Meza Torres et al., 2013a, 2013b).

La provincia del Chaco alberga al Parque Nacional Chaco, Parque Nacional El Impenetrable y a la R.N.E. Colonia Benítez, esta última adquiere importancia instructiva, ya que es utilizada como sitio didáctico por alumnos de nivel medio como también del nivel superior. Más allá de ello, ninguna de estas áreas protegidas poseen un listado de la diversidad de helechos y licofitos formalmente publicado.

Como objetivos de este trabajo se plantea brindar información sobre la diversidad de helechos y licofitos de la R.N.E. Colonia Benítez y proporcionar una clave para la determinación de los taxones presentes en el área.

\section{Materiales y Métodos}

Asignación Sistemática: Para llevar a cabo este estudio se examinaron las colecciones del Dep. Primero de Mayo, Prov. del Chaco, realizadas por el Sr. Augusto Gustavo Schulz durante casi 50 años (1929-1978), como así también especímenes de otros recolectores depositados en el herbario CTES. Además, se realizaron nuevas excursiones en distintas épocas del año que permitieron recolectar nuevos especímenes durante el año 2014. La totalidad de los ejemplares originales estudiados están depositados en el herbario CTES del Instituto de Botánica del Nordeste.

Debido a los constantes cambios en la literatura sobre la sistemática a nivel de familia en los helechos y licofitos, se presentan los resultados analizados sólo a nivel genérico e infragenérico. 

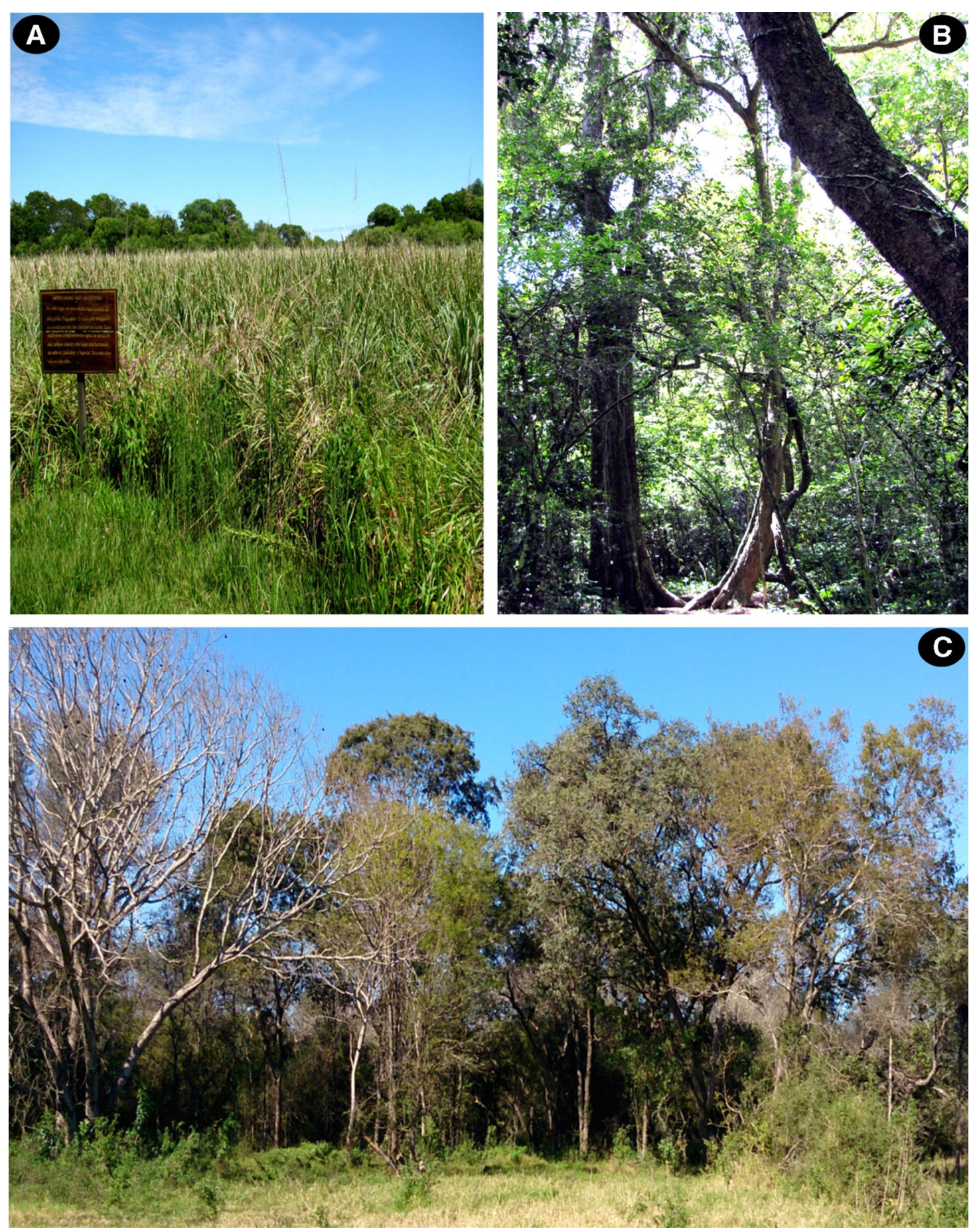

Fig. 2. Ambientes de la Reserva Natural Educativa Colonia Benítez. A: Estero. B: Relicto de selva. C: Quebrachales degradados (C, fotografía gentileza de Nicolás Niveiro).

Con los caracteres diagnósticos se confeccionó una clave dicotómica para la determinación de las especies presentes en la reserva. En el listado de taxones solo se citan dos especímenes representativos, salvo aquellos taxones que poseen un único 
registro. Todos los ejemplares citados fueron recolectados dentro de la reserva. Además se aportan datos ecológicos y otras observaciones sobre los taxones citados. La distribución geográfica de las especies para el Cono Sur de América meridional puede ser consultada en Zuloaga et al. (2008).

Análisis de la diversidad florística: Para calcular el índice de diversidad se siguió la fórmula de Squeo et al. (1998), en el cual el número de taxones depende logarítmicamente del área del lugar muestreado, y la biodiversidad taxonómica (B) se calcula como $\mathrm{B}=\mathrm{n} / \ln \mathrm{A}$, donde " $n$ " es el número de taxones registrados y " $\ln A$ " es el logaritmo natural de la superficie, aquí expresadas en ha del área en estudio.

\section{Resultados}

En la R.N.E. Colonia Benítez se registraron 17 especies reunidas en 12 géneros. El hábito terrícola fue el predominante $(70 \%)$, seguido por los epífitos facultativos (15\%), los estrictamente epífitos $(10 \%)$, y por último el hábito acuático en menor número, $(5 \%)$. Dentro del hábito terrestre se incluye a Pteris vittata L., el cual se comporta como rupícola.

Como resultado del análisis de la diversidad florística, el índice de diversidad de especies (Bsp) registrado para la R.N.E. Colonia Benítez fue de 8,17 .

Los índices de diversidad de especies de las áreas protegidas de Corrientes y Misiones fueron extraídos de Meza Torres et al. (2013b) y aquí se expresan sobre unidad de ha. Los valores son los siguientes: Reserva Cuñá Pirú (Bsp= 5,96), Reserva de Uso Múltiple Guaraní (Bsp= 9,32), Reserva Natural del Iberá $(\mathrm{Bsp}=3,14)$, Parque Nacional Mburucuyá $(\mathrm{Bsp}=4,9)$, Parque Provincial Teyú Cuaré $(\mathrm{Bsp}=5,73)$.

A continuación se presenta una clave para la determinación de los taxa de helechos y licofitos presentes en el área estudiada.

\section{Clave para la identificación de los taxa de helechos y licofitos presentes en la Reserva Natural Educativa Colonia Benítez}

1. Plantas acuáticas o palustres.

1'. Plantas terrícolas o epífitas.

2 (1). Plantas arraigadas, frondes varias veces pinnadas con indumento pruinoso

Pityrogramma calomelanos var. calomelanos

2'. Plantas flotantes, frondes bilobuladas con tricomas foliares bicelulares, a veces tricelulares. Azolla cristata

3 (1'). Plantas con licofilos linear-subulados de hasta $4 \mathrm{~mm}$ de largo, esporangios en posición adaxial y dispuestos en estróbilos de 3 a $5 \mathrm{~mm}$ de longitud. Selaginella sellowii

3'. Plantas con megáfilos, esporangios en posición abaxial, dispuestos sobre pinnas basales con láminas reducidas; soros lineares, circulares o cenosoros.

4 (3). Plantas epífitas

4'. Plantas terrícolas.

5 (4). Frondes fuertemente dimórficas, las estériles ovadas y las fértiles lanceoladas

Microgramma vaccinifolia

$5 '$. Frondes monomórficas o subdimórficas, pinnatisectas Pleopeltis minima

6 (4). Frondes palmatipartidas Doryopteris (7)

6 '. Frondes pinnadas a varias veces pinnadas

7 (6). Pecíolos con canal adaxial. Doryopteris concolor

7'. Pecíolos sin canal.

8 (7'). Pecíolos subteretes, glabros o glabrescentes; láminas con venación parcialmente reticulada, abierta en los bordes; cenosoros del segmento apical generalmente discontinuos. Doryopteris lorentzii

8'. Pecíolos teretes, raro subteretes, pubescentes, con pelos cortos castaños; láminas con venación areolada 
completa; cenosoros del segmento apical continuos

D. pentagona

9 (6'). Esporangios dispuestos en cenosoros.

Pteris (10)

9'. Esporangios dispuestos en soros circulares aislados o conniventes en la madurez, o lineares ubicados sobre venillas dicótomas.

10 (9). Láminas pinnados-pinnatífidas

Pteris denticulata var. tristicula

10'. Láminas simplemente pinnadas

Pteris vittata

$11\left(9^{\prime}\right)$. Esporangios dispuestos en soros lineares ubicados sobre venillas dicótomas

$11^{\prime}$. Esporangios dispuestos en soros circulares aislados o conniventes en la madurez.

12 (11). Láminas membranáceas, glabras Anogramma chaerophylla

12'. Láminas cartáceas, pilosas

$13\left(12^{\prime}\right)$. Esporangios dispuestos sobre las venillas en todo el envés de las pinnas. Hemionitis tomentosa

13'. Esporangios organizados únicamente en el primer par de pinnas basales modificadas. Anemia (14)

14 (13'). Pinnas fértiles insertas distanciadas de las pinnas estériles; escamas del rizoma castañorojizas. Anemia simplicior

14'. Pinnas fértiles insertas próximas a las pinnas estériles; escamas del rizoma amarillentas. Anemia tomentosa var. anthriscifolia

15 (11'). Soros en posición submarginal con pseudoindusio sin vascularización. Adiantopsis (16)

15 '. Soros en posición marginal con pseudoindusios vascularizados Adiantum lorentzii 16 (14). Láminas triangular-alargadas. Adiantopsis chlorophylla 16'. Láminas linear-lanceoladas. Adiantopsis tweedieana

\section{Listado de taxones}

Adiantopsis chlorophylla

Especímenes representativos: 7-III-2014, Dematteis 3; 21-X-1960, Schulz 11371.

Obs.: Crece en ambientes perturbados y en lugares abiertos, como bordes de senderos o en los sectores periféricos de los bosques o matorrales.

\section{Adiantopsis tweedieana}

Especímenes representativos: 23-XII-2014, Dematteis et al. 15; X-1934, Schulz 713.

Obs.: Habita frecuentemente en mosaicos de vegetación asociados con Aechmea distichantha Lem.

\section{Adiantum lorentzii}

Especimen representativo: XII-1934, Schulz 706.

Obs.: Crece en el interior de bosques y raramente sobre troncos caídos.

\section{Anemia simplicior}

Especímenes representativos: IX-1946, Schulz 6696; 2-IX-1975, Schulz 18943.

Material adicional analizado: Corrientes. Dep.
Empedrado. 8-VI-2006, Meza Torres \& LinkPérez 333 (CTES).

Obs.: Habita en pastizales o ambientes abiertos modificados, por lo general expuestos al sol. Anemia tomentosa

Especímenes representativos: 19-IX-1941, Schulz 1820; X--1946, Schulz 6494.

Obs.: Crece en el interior de bosques abiertos $\mathrm{y}$ en los bordes de senderos.

Anogramma chaerophylla

Especímenes representativos: IX/1948, Schulz 7353; 9/IX/1972, Schulz 18335.

Obs.: Habita en lugares húmedos, en pastizales, barrancas o sobre troncos en descomposición.

Azolla cristata

Especímenes representativos: 10-II-1940, Schulz 2802; VI-1950, Schulz 7568.

Obs.: Planta acuática, flotante libre, se localiza en zonas inundables y se caracteriza por formar densas poblaciones.

\section{Doryopteris concolor}

Especímenes representativos: 23-XII-2014, Dematteis et al. 13; I-1935, Schulz 720. 
Obs.: Habita dentro del sotobosque, en zonas húmedas y puede comportarse como epífito facultativo.

\section{Doryopteris lorentzii}

Especímenes representativos: 7-III-2014, Dematteis 4; 23-XII-2014, Dematteis et al. 10.

Obs.: Habita en lugares sombríos dentro del bosque, puede formar densas poblaciones, se desarrolla tanto en el suelo como en troncos en descomposición.

\section{Doryopteris pentagona}

Especímenes representativos: 23-XII2014, Dematteis et al. 11; 8-III-1972, Schulz 18074.

Obs.: Habita en interior de bosques y matorrales.

\section{Hemionitis tomentosa}

Especímenes representativos: 23-XII2014, Dematteis et al. 12; 18-X-2013, Meza Torres et al. 1550.

Obs.: Habita en el interior de bosques y puede comportarse como epífito accidental.

\section{Microgramma vaccinifolia}

Especímenes representativos: 23-XII2014, Dematteis et al. 18; X-1934, Schulz 724.

Obs.: Epífito que crece habitualmente en lugares expuestos al sol, como en los márgenes de bosques, aunque también se encuentran su interior.

\section{Pleopeltis minima}

Especímenes representativos: 7-III-2014, Dematteis 6; 4-IX-1965, Schulz 15113.

Obs.: Epífito que habita en el interior de bosques o raramente sobre ramas expuestas al sol.

\section{Pteris denticulata var. tristicula}

Especímenes representativos: 7-III-2014, Dematteis 7; I-1935, Schulz 726.

Obs.: Se localiza dentro de bosques con alta carga de humedad.

\section{Pteris vittata}

Especímenes representativos: 23-XII-2014, Dematteis et al. 16; VII-1941, Schulz 3050.
Obs.: Especie adventicia con hábito rupícola, raro terrestre, crece sobre paredones húmedos por lo general en ambientes urbanos o suburbanos.

Pityrogramma calomelanos var. calomelanos

Especimen representativo: 23-XII-14, Dematteis et al. 17.

Obs.: Habita en lugares bajos, en pastizales que rodean lagunas y bañados, e incluso en embalsados.

Selaginella sellowii

Especímenes representativos: 23-XII-2014, Dematteis et al. 14; IV-1937, Schulz 366.

Obs.: Crece habitualmente en terrenos erosionados, habitualmente con algún grado de anegamiento.

\section{Discusión y Conclusiones}

Del análisis del inventario realizado, se desprende que los tres géneros que se encuentran mejor representados con especies nativas son Doryopteris J. Sm., Adiantopsis Fée y Anemia Sw. El primero de ellos presenta tres especies, D. pentagona Pic. Serm., D. concolor (Langsd. \& Fisch.) Kuhn y $D$. lorentzii (Hieron.) Diels. El género Adiantopsis registra dos especies, A. chlorophylla (Sw.) Fée y A. tweediana (Hook.) Link-Pérez \& Hickey, al igual que Anemia Sw., [A. simplicior (H. Christ) Mickel (Fig. 3A) y $A$. tomentosa (Savigny) Sw. var. anthriscifolia (Schrad.) Mickel], y Pteris L., [P. denticulata Sw. var. tristicula (Raddi) J. Prado (Fig. 3B) y P. vittata].

Los demás géneros presentaron sólo una especie, Adiantum lorentzii Hieron., Anogramma chaerophylla (Desv.) Link, Azolla cristata Kaulf., Hemionitis tomentosa (Lam.) Raddi (Fig. 3 C-D), Microgramma vaccinifolia (Langsd. \& Fisch.) Copel., Pityrogramma calomelanos (L.) Link var. calomelanos, Pleopeltis minima (Bory) J. Prado \& R.Y. Hirai (Fig. 3E) y Selaginella sellowii Hieron.

De los taxones antes mencionados, Pteris vittata y Pityrogramma calomelanos var. calomelanos, fueron recolectados dentro del predio del INTA que rodea a la R.N.E. Colonia Benítez, a una distancia aproximada de $100 \mathrm{~m}$. 

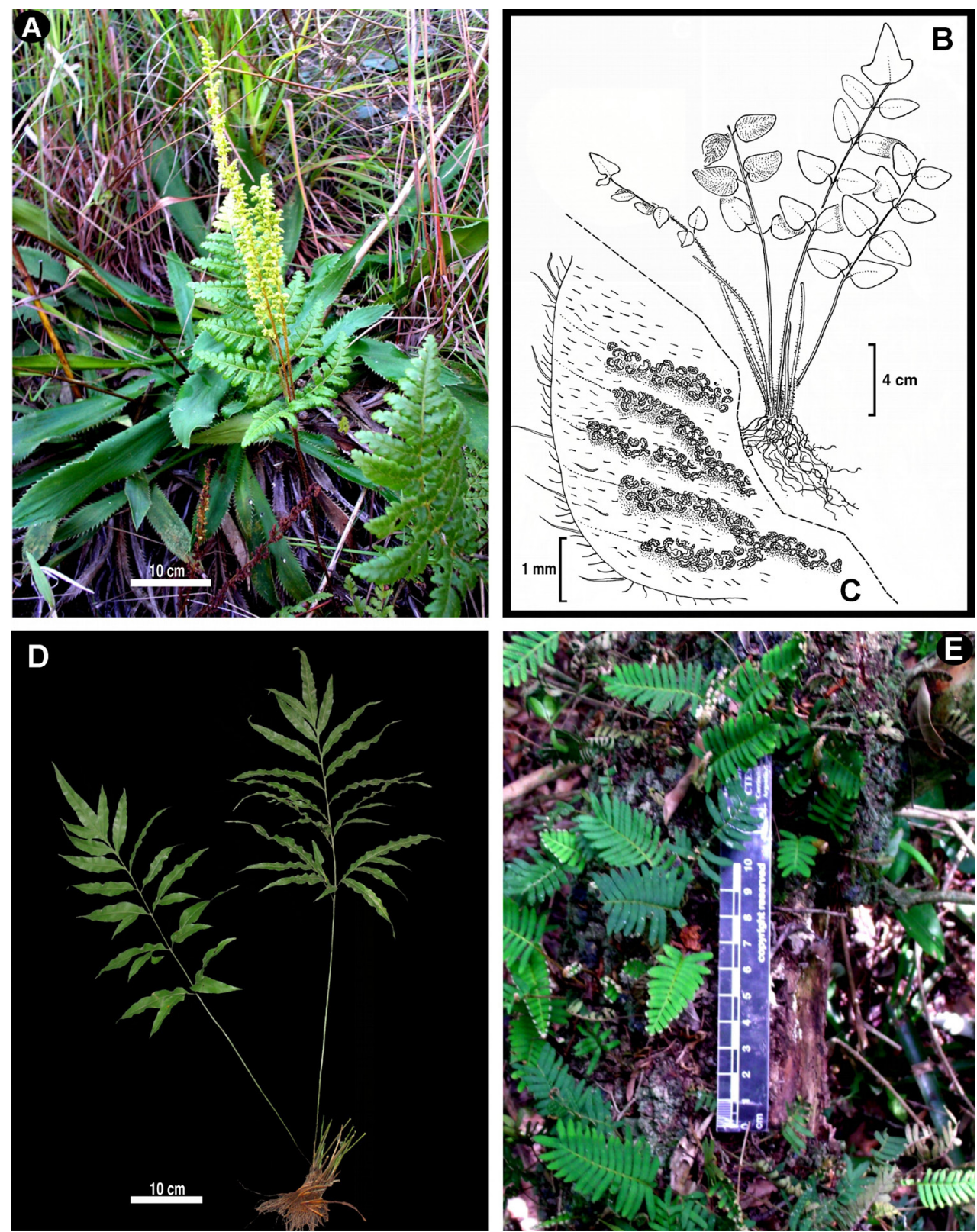

Fig. 3. Helechos de la Reserva Educativa Colonia Benítez. A: Anemia simplicior. B: Pteris denticulata var. tristicula. C-D: Hemionitis tomentosa. C: Porte. D: Detalle de la disposición de esporangios. E: Pleopeltis minima. (A: Meza Torres \& Link-Pérez 333; B-C: Dematteis et al. 12; D: Dematteis 7; E: Dematteis 6).

En su totalidad se registraron en la R.N.E. Colonia Benítez 12 géneros y 17 taxones infragenéricos de helechos y licofitos, los cuales representan aproximadamente el 30\% de la diversidad de estos grupos vegetales citados para la provincia del Chaco.

Si se compara con los índices de diversidad en especies de helechos y licofitos de las demás áreas protegidas del noreste argentino [Reserva Cuñá Pirú (Bsp= 5,96), Reserva de Uso Múltiple Guaraní $(B s p=9,32)$, Reserva Natural del Iberá $(\mathrm{Bsp}=3,14)$, Parque Nacional Mburucuyá $(\mathrm{Bsp}=$ $4,9)$, Parque Provincial Teyú Cuaré $(\mathrm{Bsp}=5,73)]$, la R.N.E. Colonia Benítez adquiere importancia 
debido a que posee un $\mathrm{Bsp}=8,15$. Este índice ubica al área aquí estudiada en segundo término dentro de las reservas del noreste argentino registradas en la literatura, solo superada por la Reserva de Uso Múltiple Guaraní.

Colonia Benítez se encuentra dentro de la influencia del corredor biogeográfico fluvial Paraná-Paraguay, más precisamente en el trecho Paraná Medio (Oakley et al., 2005). Este corredor biogeográfico se evidencia en que todos los bosques en galería presentes en este sistema fluvial poseen linaje amazónico, llegando incluso hasta los denominados 'Montes Blancos' del Delta del Paraná (Oakley et al., 2005). De este modo se explica la presencia de taxones amazónicos, como Hemionitis tomentosa y Pteris denticulata var. tristicula en Colonia Benítez, dentro de lo que Cabrera (1971) consideró Dominio Chaqueño. Otras especies como Doryopteris concolor, D. pentagona y Pleopeltis minima al parecer también usan este corredor biogeográfico para llegar hasta los bosques de Punta Lara, sobre el margen del Río de La Plata, como lo registraron Giudice et al. (2011).

Por lo expuesto anteriormente, se valoriza la propuesta impulsada en 1965 por A.G. Schulz de crear un área protegida en esta localidad, con el fin de conservar su gran patrimonio biótico. Este tipo de medidas favorecen la conservación in situ de la biodiversidad regional y concede al área un alto valor didáctico para la enseñanza de botánica y ecología en los distintos niveles de la educación.

\section{Agradecimientos}

Los autores agradecen al cuerpo de Guardaparques de la R.N.E. Colonia Benítez por su constante atención y predisposición para ayudar a realizar el trabajo; a Walter Medina quién brindó su ayuda en los viajes de campaña; a Francisco Squeo Porcile por responder todas las consultas; a María Silvia Ferrucci por la lectura crítica del manuscrito. Trabajo subsidiado por la Secretaría General de Ciencia y Técnica de la Universidad Nacional del Nordeste (SGCyTUNNE. PI N A012-2013.)

\section{Bibliografía}

ARBO, M. M. \& S. G. TRESSENS. 2002. Flora del Iberá. EUDENE-UNNE Corrientes, Argentina.

BIGANZOLI, F. \& M. E. MÚLGURA DE ROMERO. 2004. Inventario Florístico del Parque Provincial Teyú Cuaré y alrededores (Misiones, Argentina). Darwiniana (Argentina) 42: 1-24.

CABRERA, A. L. 1971. Fitogeografía de la República Argentina. Bol. Soc. Argent. Bot. 14: 1-42.

DE LA SOTA, E. R. 1977. Pteridophyta. En: A. Cabrera (ed.). Flora de Jujuy. Inst. Nac. Tecnol. Agropecu. 13: 1-275.

GIUDICE, G. E., J. P. GIACOSA RAMOS, M. L. LUNA, A. A. YAÑEZ \& E. R. DE LA SOTA. 2011. Diversidad de helechos y licofitas de la Reserva Natural Punta Lara, Buenos Aires, Argentina. Rev. Biol. Trop. 59: 1037-1046.

MÁRQUEZ, G. J., G. E. GIUDICE \& M. M. PONCE. 2006. Pteridofitas de la Reserva "Valle del Arroyo Cuñá Pirú" (Misiones, Argentina). Darwiniana 44: 108-126.

MEZA TORRES, E. I., E. R. DE LA SOTA \& M. S. FERRUCCI. 2013a. Sinopsis de los helechos y licofitos del Parque Nacional Mburucuyá, Corrientes, Argentina. Bol. Soc. Argent. Bot. 48: 121-136.

MEZA TORRES, E. I., E. R., DE LA SOTA \& M. S. FERRUCCI. 2013b. Biogeographic analysis and key to genera of ferns and lycophytes of Mburucuyá National Park, Corrientes, Argentina. Rev. Chil. Hist. Nat. 86: 49-61.

OAKLEY, L. J., D. PRADO \& J. ADAMOLI. 2005. Aspectos biogeográficos del corredor fluvial Paraguay-Paraná. En: F. Aceñolaza (ed.), Temas de la Biodiversidad del Litoral 14: 245-258. Magna, San Miguel de Tucumán.

PONCE, M., K. MEHLTRETER \& E. R. DE LA SOTA. 2002. Análisis biogeográfico de la diversidad pteridofítica en Argentina y Chile continental. Rev. Chil. Hist. Nat. 75: 703-717.

SQUEO, F. A., L. A. CAVIERES. G., ARANCIO, J. E. NOVOA \& O. MATHEI. 1998. Biodiversidad de la Flora Vascular en la Región de Antofagasta. Rev. Chil. Hist. Nat. 71: 571-591.

TRESSENS, S. G., H. KELLER \& V. REVILLA. 2008. Las plantas vasculares de la reserva de uso múltiple Guaraní, Misiones, Argentina. Bol. Soc. Argent. Bot. 43: 273-293.

ZULOAGA, F. O., O. MORRONE \& M. BELGRANO (eds.). 2008. Catálogo de las plantas vasculares del Cono Sur. (1). Monogr. Syst. Bot. Missouri Bot. Gard. 107: 1-161.

Original recibido el 4 de marzo de 2015; aceptado el 6 de abril de 2015. 
